\title{
Human immunodeficiency virus: Fragments of the puzzle
}

IN THIS ISSUE OF THE JOURNAL, TWO IMPORTANT PAPERS I(pages 290, 295) describing the progress of the human immunodeficiency virus (HIV) epidemic in Canada are published. Changes in the demographics of this disease in the United States are well- described and document a steady shift to less advantaged groups in society including intravenous drug abusers, individuals in the sex trade and certain members of the immigrant population. The fragmentary data available to us from epidemiological studies in Canada suggest that the shift in Canada is not as marked as that observed in the United States, but the expectation is that similar trends will be observed. Our knowledge of the extent of infection and impact of this virus in Canada, however, is described poorly. What do we know about the current status of this most important public health problem at the end of the 20th century?

The paper by Ricketts et al, from the Laboratory Centre for Disease Control, is reassuring. A surveillance system enrolling subjects from across Canada to follow significant occupational exposure to HIV has failed to document prospectively any case of transmission of the virus occupationally in Canada after six and one-half years. One recent anecdotal report (1) does suggest possible occupational acquisition by a technologist early in the epidemic, before current programs to prevent transmission were in place and before this surveillance program was initiated. Studies from the United States (2) have documented that the risk of acquiring HIV infection following needlestick exposure to a known infected patient is 0.3 to $0.5 \%$. The risk likely is greater when the exposure allows inoculation of greater quantities of blood. This low rate of transmission, compared with agents such as hepatitis B, reflects the low inoculum of infectious virus present in the blood of most individuals infected with HIV. Given these reported transmission rates it is not surprising - with the numbers of episodes enrolled in the Canadian Surveillance Study - that an episode of prospectively documented occupational transmission in Canada has not been observed. The study confirms, however, that in
Canada, as elsewhere, the occupational risk of transmission of HIV is extraordinarily small. This is reassuring to all of us who work in health care. The introduction of needleless devices and other programs to limit exposure to sharps and potential injuries hopefully will further enhance the safety of the hospital and clinic environment.

The study by Sekla et al from Manitoba is much less reassuring; it is one of the first published unlinked seroprevalence studies in a Canadian population. This study confirms the impression from acquired immune deficiency syndrome incidence reports of Manitoba as a low prevalence area for HIV infection. The prevalence of HIV infection in screening of prenatal bloods was only 0.72 per 10,000 compared with British Columbia (2.7 per 10,000) or Quebec (6.1 per 10,000). This study also suggests, for Manitoba, that HIV infection is not yet common in the parenteral drug abuser or prostitute population.

What is surprising, however, is that even for a geographic area of low prevalence for HIV infection, the rate among men in Manitoba assessed for sexually transmitted diseases was 143 per 10,000. As the authors point out, this identifies a subgroup of individuals who should be targeted for screening programs. Similar studies reporting observations from other provinces across Canada are essential to map the full picture of HIV infection in this country. Repeated studies will be one means of monitoring the progress of the epidemic.

Recommendations for HIV screening have consistently prompted intense controversy. Early in the epidemic, there were arguments for not screening based on human rights concerns, validity of laboratory tests and questions with respect to the individual benefit for an asymptomatic subject to know her or his serological status. The last two items are no longer issues. Diagnostic testing is reliable and options for current management make it clear that early identification of infected individuals is not only desirable, but essential. Provision of early antiretroviral therapy and 
prophylaxis for important infections such as Pneumocytis carinii pneumonia improves the quality of life and prolongs survival for HIV-infected subjects. Thus, there are strong medical arguments for screening of high prevalence populations. There are also public health arguments. Identification of individuals infected with HIV should allow the opportunity to educate them with respect to transmission and behaviour modification to prevent transmission. While this may not always be effective, early identification of infection and contact tracing must be a primary method of control.

These arguments for more intensive screening of populations with a high prevalence of HIV infection are compelling. In Manitoba, one group who should be encouraged to accept screening would be males being assessed for sexually transmitted diseases. High prevalence groups in other parts of the country similarly should be identified. Some elements of our society may still penalize individuals found to be HIV-infected, and individual rights must be protected. However, a more 'routine' approach to identification of HIV infection may help to demystify this disease and ameliorate some public misconceptions.

The goals we have with respect to managing HIV infection in Canada are straightforward. First, we wish to prevent transmission of the infection to noninfected individuals. While programs of education and screening may have some impact on this goal, success realistically requires development and widespread use of an effective vaccine. Second, we wish to cure individuals infected with this virus. This currently is not achievable. Further intensive basic research to understand the virus and its biology, and to accelerate the development of antiviral therapies will be necessary to achieve this goal. Third, if we cannot cure the infection, we wish to optimize medical care for infected individuals. This requires ongoing clinical trials and assessments of new therapeutic, preventive and suppressive therapies. Appropriate medical facilities to optimize outpatient management and provision of palliative care are part of this goal. Underpinning all these efforts must be high quality, current, epidemiological data describing the progress of this epidemic in Canada and analyzing, on an ongoing basis, the changes occurring. The studies of Ricketts el al and Sekla et al are important and helpful, but much further information is required.

\section{REFERENCES}

1. Health and Welfare Canada. A case of HIV infection possibly transmitted in an occupational setting - Ontario. Canada Communicable Disease Report 1992:18:13.

2. United States Department of Health and Human Services, Centers for Disease Control. Guidelines for prevention of transmission of human immunodeficiency virus and hepatitis B virus to health care and public safety workers. MMWR 1989;38(S-6): 1-38.

LE Nicolle, MD

Editor-in-Chief

Winnipeg, Manitoba 


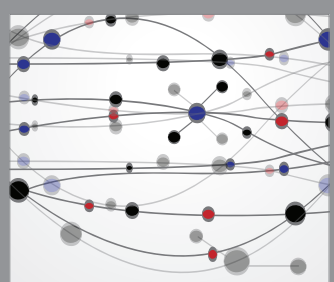

The Scientific World Journal
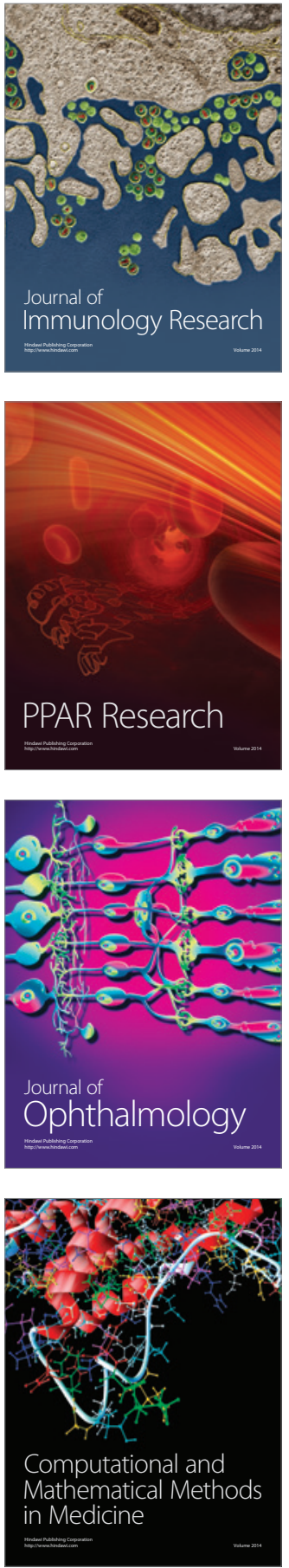

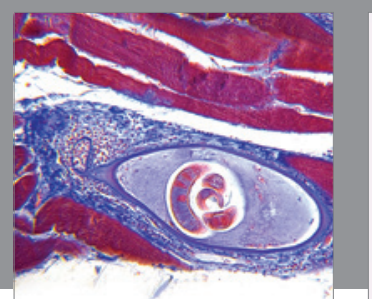

Gastroenterology Research and Practice

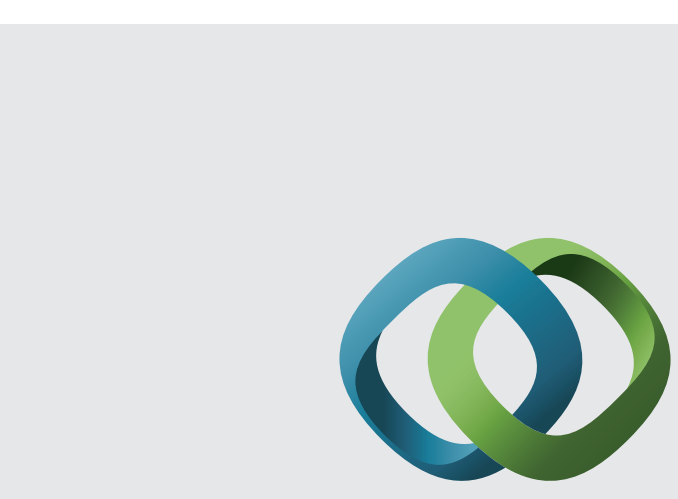

\section{Hindawi}

Submit your manuscripts at

http://www.hindawi.com
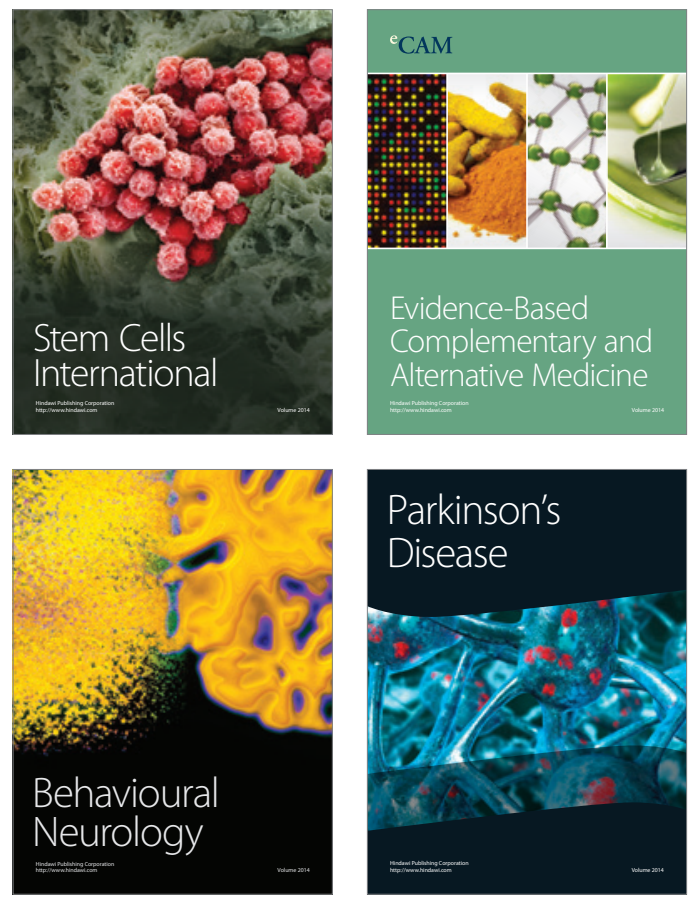
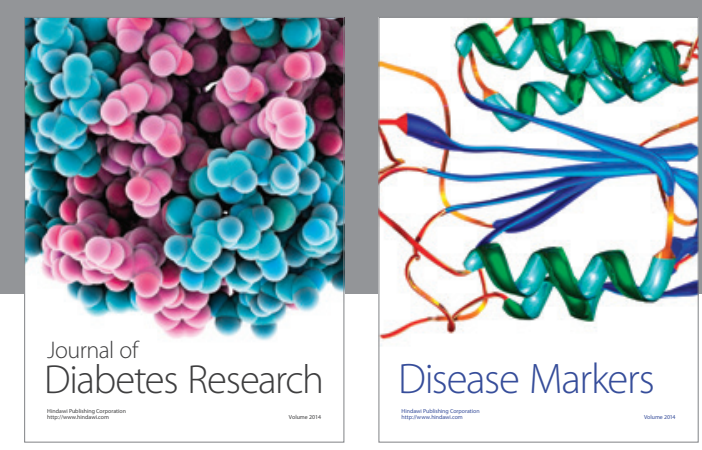

Disease Markers
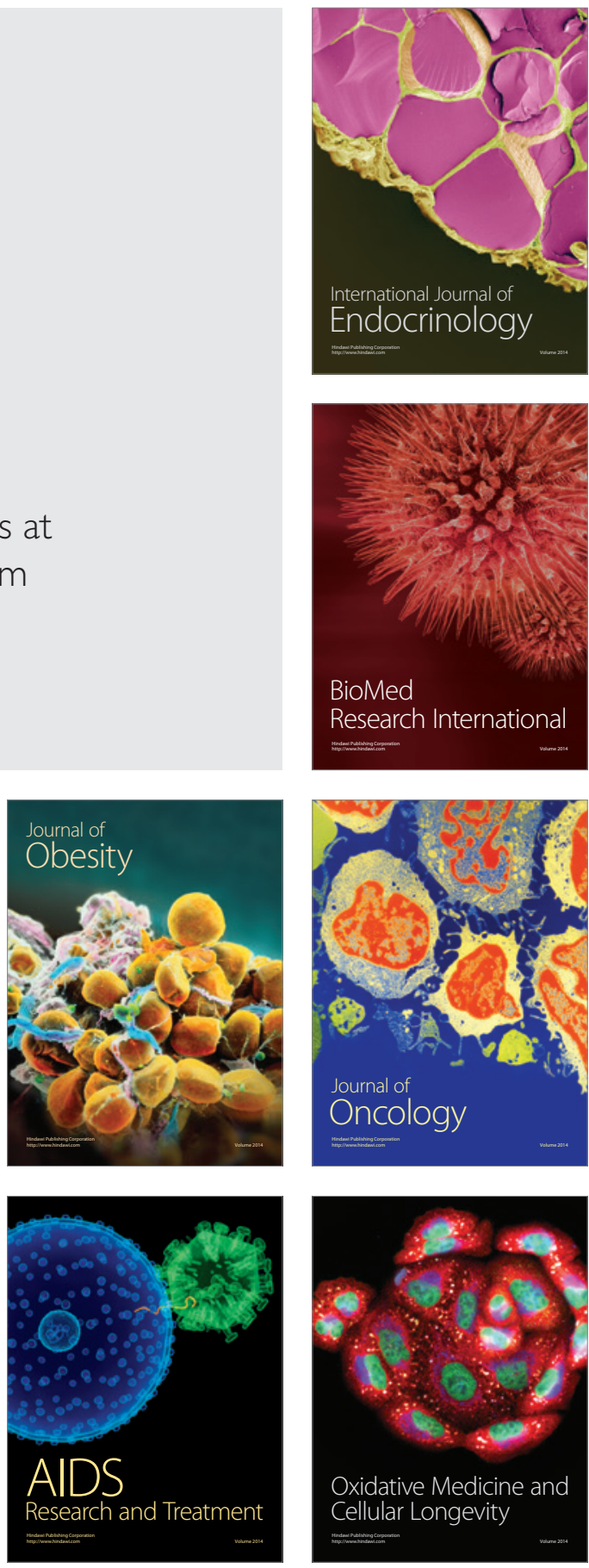